\title{
A ROBUST DEEP LEARNING APPROACH TO ENHANCE THE ACCURACY OF POMEGRANATE FRUIT DISEASE DETECTION UNDER REAL FIELD CONDITION
}

\author{
Vaishali Nirgude", Sheetal Rathi
}

Department of Computer Engineering, Thakur College of Engineering and Technology, Mumbai, India

Received - August 19, 2021; Revision - November 10, 2021; Accepted - December 19, 2021

Available Online - December 30, 2021

DOI: http://dx.doi.org/10.18006/2021.9(6).863.870

\author{
KEYWORDS \\ Deep Learning \\ Convolution Neural Network \\ Pomegranate \\ Agriculture \\ Disease Management
}

\begin{abstract}
Pomegranate fruits are infected by various diseases and pests, which negatively affect food security, productivity, and quality. Recent advancements in deep learning with Convolutional Neural Networks (CNNs) have significantly improved the accuracy of fruit disease detection and classification. The main objective of this investigation is to find the most suitable deep-learning architecture to enhance fruit disease detection and classification accuracy. The current study proposed an efficient deep learningbased approach to detect the most prominent diseases of pomegranate such as bacterial blight, anthracnose, fruit spot, wilt, and fruit borer. For experimentation, a total of 1493 stagewise diseases development images of fruits and leaves are captured via a camera of an interval of 25 days for a total of six months duration. Additionally, extensive data augmentation was performed to increase the dataset, data diversity and to achieve a more robust model for disease detection. For this, the performance of three CNN-based architectures i.e., ResNet50, ResNet18, and Inception-V3 on a real field environment dataset was measured. Experimental results revealed that the proposed CNN-based ResNet50 architecture has effectively detected and classified five different types of diseases whose symptoms are not well defined and with the capability to deal with complex backgrounds. The optimized ResNet50 model achieved $97.92 \%$ test accuracy over ResNet18 (87.5\%) and Inception-V3 (78.75\%) on learning rate 0.001 . The multiclass cross-entropy loss function is applied for determining the error rate. To deal with CNN 'Black Box' problem Grad-CAM model can be used in the future. The proposed method will help the agricultural industry in detecting the most prominent diseases of pomegranate, which are likely to cause a decrease in productivity, thereby avoiding economic loss.
\end{abstract}

* Corresponding author

E-mail: vaishali.nirgude@thakureducation.org (Vaishali Nirgude)

Peer review under responsibility of Journal of Experimental Biology and Agricultural Sciences.

Production and Hosting by Horizon Publisher India [HPI] (http://www.horizonpublisherindia.in/).

All rights reserved.
All the articles published by Journal of Experimental Biology and Agricultural Sciences are licensed under a Creative Commons Attribution-NonCommercial 4.0 International License Based on a work at www.jebas.org.

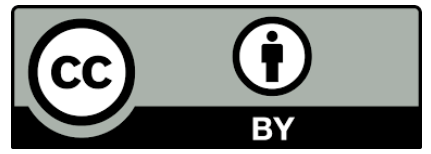




\section{Introduction}

Agriculture plays an inherent part in the Indian economy (Reddy \& Dutta, 2018). According to the Food and Agricultural Organization of the United Nations (FAO-UN), 70\% of people are depending on agriculture resources and allied agricultural products in India (OECD/FAO, 2016). Fruit disease is one of the major hazards to food safety (Rehman et al., 2021). According to the Technology Information, Forecasting and Assessment Council (TIFAC), 18\% of fruit loss is due to various pests and diseases, while $25-30 \%$ of the overall production is lost due to wastage at post-harvest stages. When the disease is spread throughout the farm, it may result in noteworthy loss both in quality as well as the number of fruits. The prominent reason behind farmer suicide is crop failure and it is $11.2 \%$ of all suicides in India (Hossain et al., 2020). Thus, accurate and timely detection and prediction of fruit diseases will be helpful to farmers, customers, and the government for making strategies such as trade-offs, pricing, storage, and food shortage relief. Generally, fruit diseases are detected manually, which is time-consuming and continuous monitoring of the field is required by the expert, which proves costly. Additionally, the expert should be available for consultation whenever symptoms appear. Fruit diseases can be tested in the laboratory however, it requires satisfactory laboratory conditions along with professional domain expert's knowledge. Therefore, automatic early fruit disease detection and suitable prevention measures should be identified first to get rid of disease and secondly to minimize the spread of diseases. It will save our food donors and improve the economic development of the nation.

Pomegranate ( $P$. granatum) is one of the commercial fruits and is mostly cultivated in arid and semiarid regions in India. The fruits are extremely important as they are delicious and rich in carbohydrates, calcium, iron, vitamin-c, and citric acid (MelgarejoSánchez et al., 2021). It has high medicinal value, therefore, helps to cure diabetes, cancer, hypertension, heart, and kidney diseases (Kandylis \& Kokkinomagoulos, 2020). India stands first for its pomegranate fruit production of around 5 lakh tons per year. Maharashtra is the leading state with 43 thousand hector areas under pomegranate cultivation followed by Karnataka, Gujrat, and Andhra Pradesh (Yadav et al., 2020). Nowadays, due to sudden changes in climate fruits get affected by various known and unknown diseases which results in huge losses to the farmers. Study shows that less research work has been carried out on real field pomegranate fruit diseases management. This study was carried out to real field environment pomegranate disease detection using DL models. The proposed system helps the farmers to detect diseases and recommend appropriate treatment and preventive measures to avoid losses.

DL approach is a subcategory of ML, introduced in 1943. The recent structures of DL are Deep Neural Networks (DNN),
Convolutional Neural Networks (CNN), Recurrent Neural Networks (RNN), and Generative Adversarial Networks (GAN), which has been widely studied and applied in various fields including agriculture (Sarker, 2021). Among these Convolution Neural Networks (CNNs) have achieved better accuracy in the detection and classification of various fruits diseases over image processing (Hassan et al., 2021). There are total of $15-\mathrm{CNN}$ based architectures that are trained on the ImageNet dataset consist 1000 classes and 1.2 million images (Deng et al., 2009). ImageNet Large Scale Visual Recognition Challenge (ILSVRC) aimed to evaluate algorithms for object detection and image classification (Russakovsky et al., 2015). CNN-based architecture AlexNet (60M parameters, 8 layers, first to implement ReLu function) achieved a top-5 error rate (13.5\%) and won the ILSVRC-2012 competition (Krizhevsky et al., 2012). In 2013, a variation of AlexNet called ZFNet (Clarifai) has won the ImageNet classification task (Zeiler \& Fergus, 2014). GoogLeNet Architecture (5M parameters, factorized 7 X 7 Convolutions, 22-layers, Inception module, Computational power) was the winner of ILSVRC 2014 having an error rate of $6.7 \%$ for object classification and detection (Szegedy et al., 2016). In 2014, VGG (138 M parameters, 19 layers) has achieved better accuracy on ILSVRC classification and localization tasks (Simonyan \& Zisserman, 2015). ResNet architecture (Residual Network with skip connection, 152 layers, overcome vanishing and exploding gradient problem) won the 2015 ImageNet competition in both classification and detection tasks with only $3.57 \%$ error rate and $96.4 \%$ accuracy (He et al., 2015).

The objective of this study is to compare widely used CNN-based architectures to improve pomegranate disease detection accuracy and reduce the loss. The proposed system helps farmers to detect diseases and recommend appropriate treatment and preventive measures to avoid losses.

\section{Materials and Methods}

\subsection{Research Methodology}

The proposed system is based on a deep learning model. It consists of five units as follows: Data Collection, Data Pre-processing, Model Generation, Research Outcome, and Data Visualization as shown in figure 1.

\subsection{Fruit Disease Management and Data Preparation for Experimentation}

Fruit disease management is essential to improve the quality and quantity of fruits. Pomegranate fruits disease management includes disease classification, detection, prediction, disease treatment, and preventive measures (Yadav et al., 2020). Different varieties of pomegranate fruits are available in Maharashtra. For this study, we 


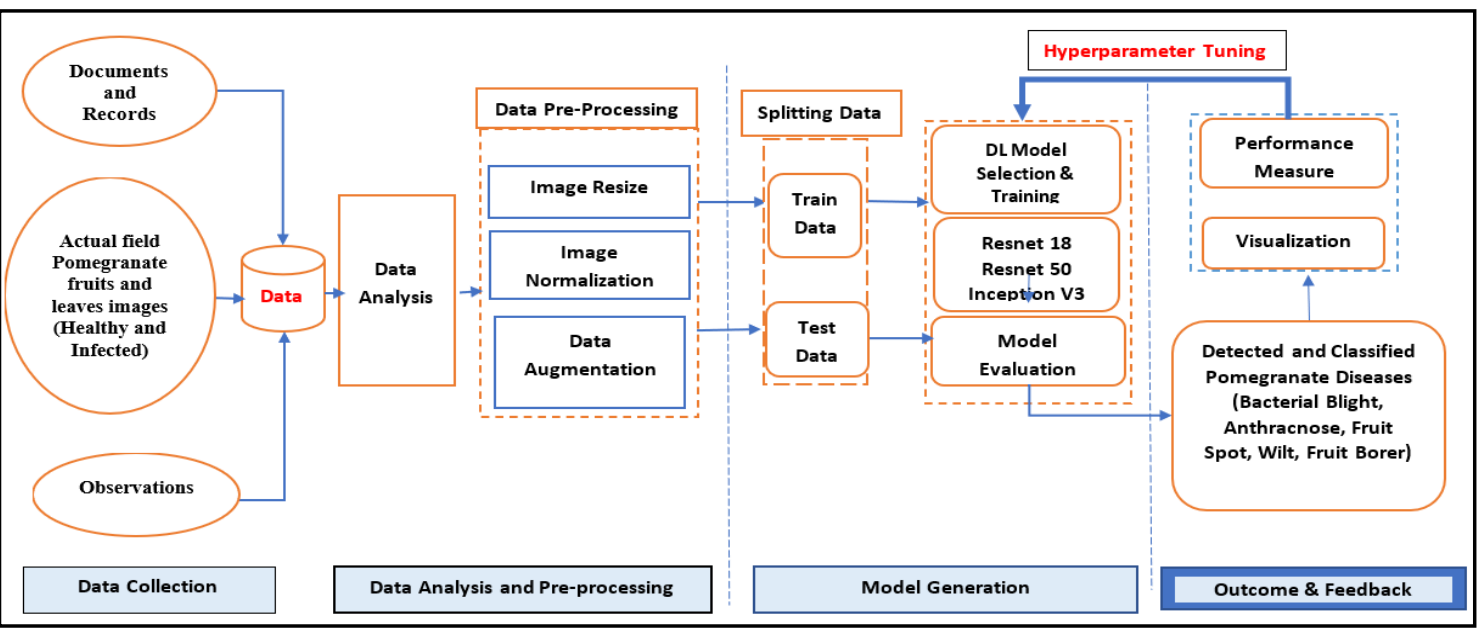

Figure 1 Proposed System Architecture for Fruit Disease Detection

have collected real field images of the Ganesh variety. Open benchmark pomegranate fruits dataset is not available. Therefore, for experimentation image dataset is obtained by visiting five different pomegranate orchards in Pathare Village, Nashik district, Maharashtra. Stagewise diseases development images are captured via a camera at an interval of 25 days for a total of six months duration. Figure 2 shows sample basis images. Quality data collection and its pre-processing is one of the crucial steps along with the appropriate model selection to enhance the disease detection accuracy. Total 1493, real field pomegranate fruits and leaf images of the most prominent diseases are collected using a camera. These images have been classified into six categories: Healthy, bacterial blight, anthracnose, fruit spot, wilt, and fruit borer. All the collected images are varied in size therefore data preprocessing has been performed. First, input images are resized (224 X 224 X 3) to match the size of the input layer of the $\mathrm{CNN}$-based architecture. Secondly, images are normalized (Batch Normalization) to help the model train faster and to better generalize on unseen data (Wang et al., 2021). Data augmentation is the process of transformation of the geometry or intensity of the original images to make new images (Taylor \& Nitschke, 2018). Dynamic data augmentation has been done to increase the size of the dataset and its diversity. Data augmentation also helps to solve unbalanced data and make the model more robust (Shorten \& Khoshgoftaar, 2019). Data augmentation is done by rotation (flip horizontal, vertical), random cropping, zooming, changing contrast or brightness, removal of background, and adding noise.
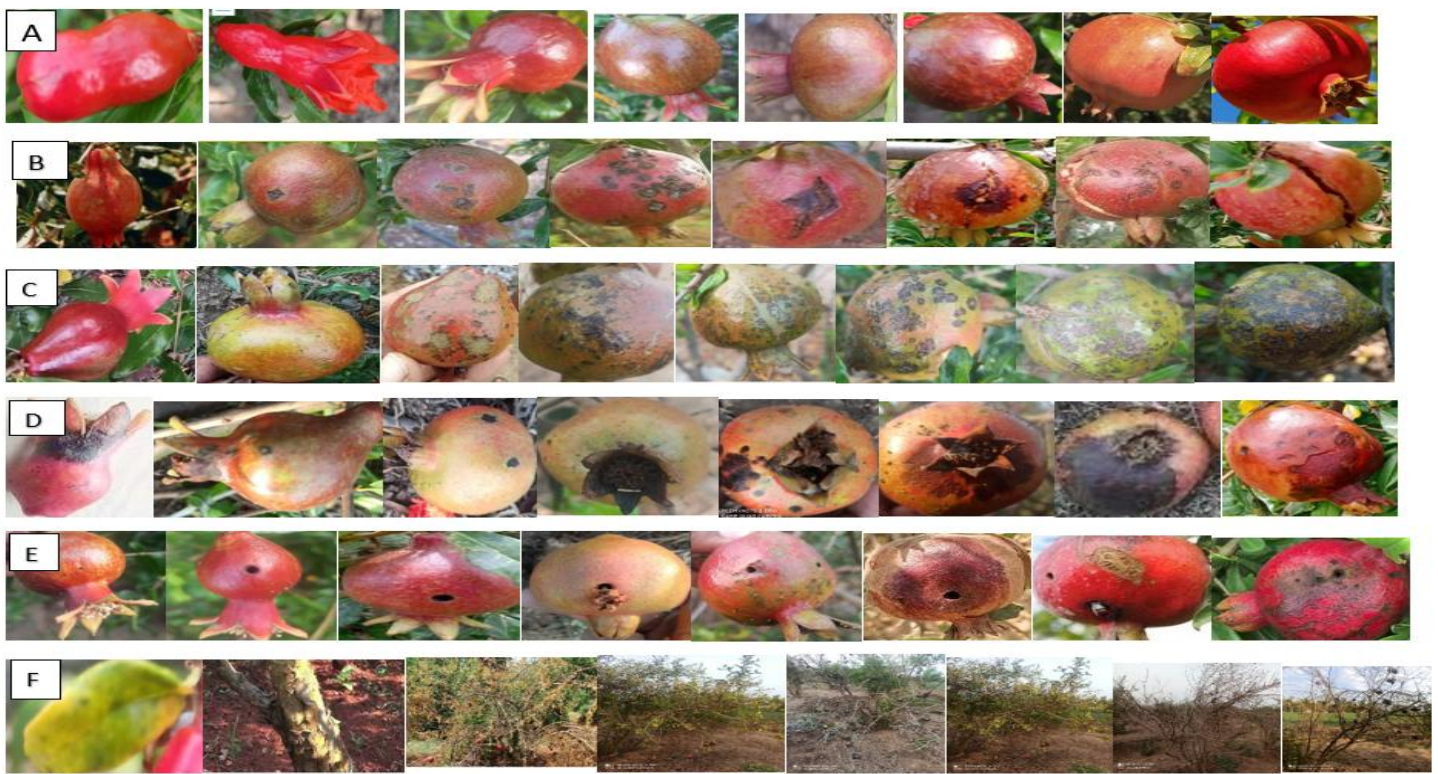

Figure 2 Sample stagewise healthy and disease development images of Pomegranate fruits collected from real field environment of variety Ganesh (A) Healthy fruit, (B) bacterial blight (Telya in Marathi), (C) Anthracnose, (D) Fruit Spot, (E) Fruit Borer, and (F) Fusarium Wilt.

Journal of Experimental Biology and Agricultural Sciences http://www.jebas.org 


\subsection{Deep Learning Model Selection and Training}

Out of total 15 different pre-trained architectures based on the CNN model, ResNet18, ResNet50, and Inception-V3 architectures were trained on a pomegranate image dataset for disease detection. The proposed model was implemented using Python Programming (https://www.python.org) with the PyTorch framework (https: //pytorch.org).

\subsubsection{Proposed CNN Based ResNet50 Architecture}

ResNet18 and ResNet50 are trained on the ImageNet dataset. The baseline structure of both is the same except the bottleneck layer has been added in ResNet50 for dimensionality reduction. DL models suffer from vanishing gradient or exploding gradient problems (Liu et al., 2021). Residual network (skip connection) is added in ResNet architecture to overcome the vanishing and exploding gradient effect. ResNet model developed by Microsoft with $18,34,50,101, \& 152$ layers (He et al., 2015). ResNet18 consists of a residual block while ResNet50 consists of a bottleneck layer. The residual network has two building blocks: a Base block and a Bottleneck block. The base block consists of 2 convolution layers, activation function (ReLu), and batch normalization whereas, the Bottleneck block consists of 3 convolution layers. Figure 3 represents the proposed architecture of ResNet50 for pomegranate fruit disease detection. The first layer has a $7 * 7$ filter size and 64 such filters with a stride of 2 . The first convolutional layer consists of a filter size of $1 * 1$ with 64 such filters, the next layer with a filter size of $3 * 3$ and 64 such filters. Further layer with $1 * 1$ filter size and 256 such filters. A similar combination has been repeated 2 more times to get 3 such residual layers and in turn 3 layers in each layer as a result of 9 such layers. Similarly with a certain combination of filter size and filters in the arrangement of 4,6,3 layers. Thus, a total number of layers= $3 * 3+4 * 3+6 * 3+3 * 3=50$. Skip connection with the help of Identity block is used to provide the value of the input to the output layer. The size of the image from one layer to another layer changes with the following formula:

$$
\mathrm{S}=\frac{\mathrm{n}+2 * \mathrm{P}-\mathrm{f}}{\mathrm{s}}+1 * \frac{\mathrm{n}+2 * \mathrm{P}-\mathrm{f}}{\mathrm{s}}+1
$$

Whereas $\mathrm{n}=$ pixel size, $\mathrm{p}=$ padding and $\mathrm{f}=$ filter size and $\mathrm{s}=$ stride. The experimental result proves that ResNet50 gives better fruit disease detection accuracy than other networks.
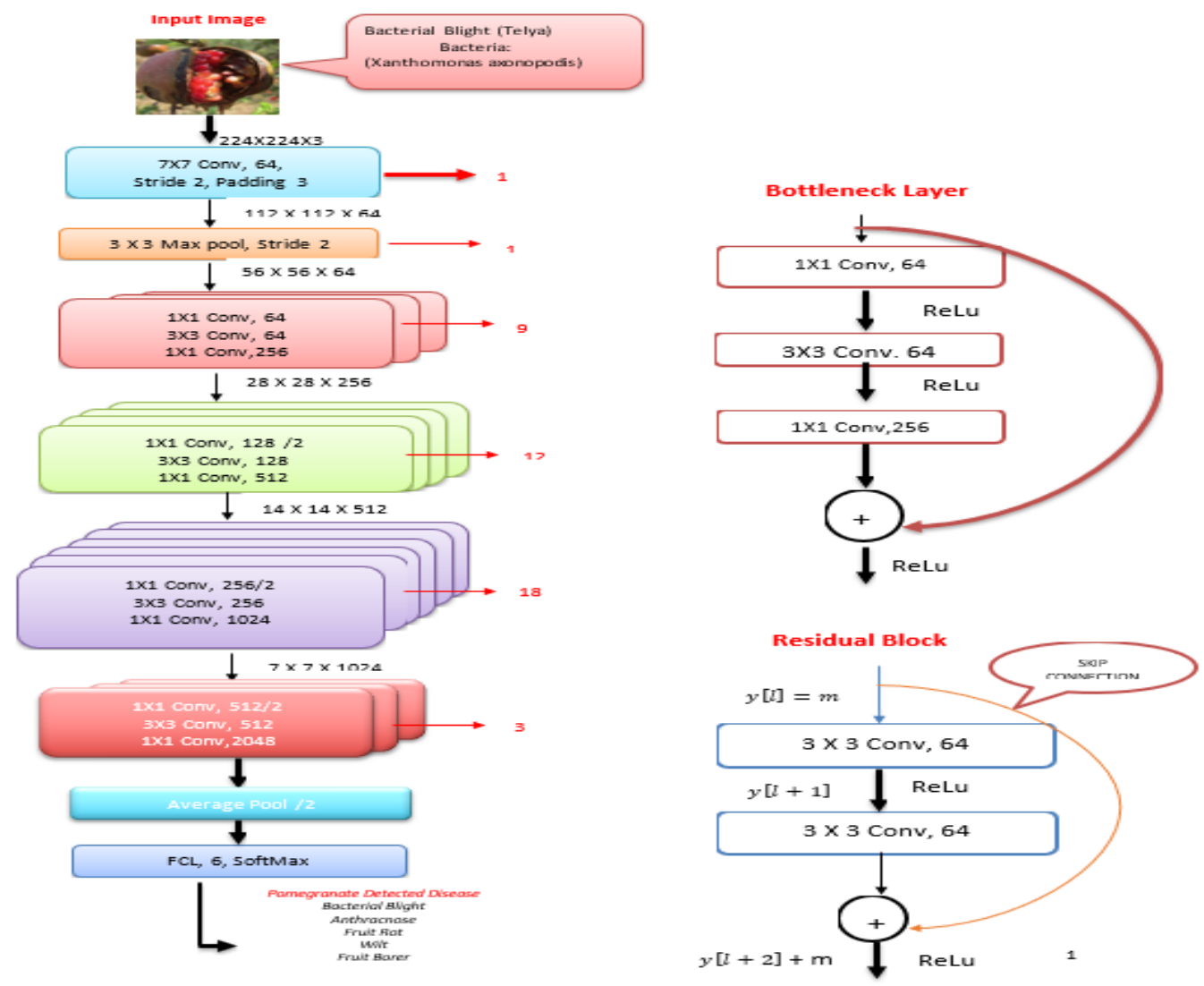

Figure 3 Proposed ResNet50 architecture for pomegranate fruit disease detection

Journal of Experimental Biology and Agricultural Sciences http://www.jebas.org 


\subsubsection{Mathematical Equation for Residual Network}

Residual Network works on the concept of skip connection i.e., "Adding the original input to the output of convolutional block". Hence by skipping certain layers, the value of input can be avoided to be reaching to extreme values (Either very small or very large) (He et al., 2015).

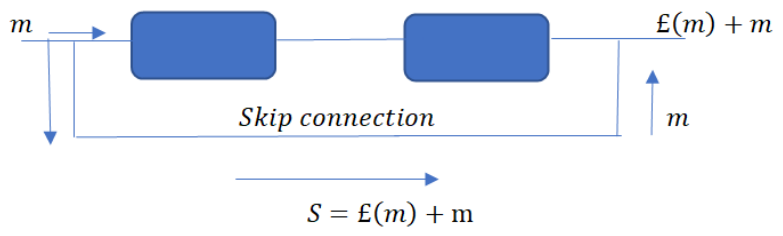

Whereas $S$ is the output, $m$ is the input, and $f(m)$ is the function of input i.e., $\mathrm{m}$.

Output of Layer l : $\quad \mathrm{y}[\mathrm{l}]=\mathrm{m}$

Output of Layer l+1: $\quad Z[l+1]=w[l+1] . y[l]+b[l+1]$

Apply ReLu activation function.

$$
y[l+1]=\operatorname{relu}(Z[l+1])
$$

Output of Layer l+2: $z[l+2]=w[1+2] \cdot y[l+1]+b[1+2]$ (iv)

Apply ReLu activation function

$$
y[1+2]=\operatorname{relu}(\mathrm{Z}[1+2])
$$

Add Skip connection and Rewrite eq. [V]

$$
y[1+2]=\operatorname{relu}((z[1+2])+y[1])
$$

L2-Regularization makes, $w[l+2]=0, b[l+2]=0$, so itwill make

$$
\begin{gathered}
\mathrm{Z}[\mathrm{l}+2]=0 \\
y[l+2]=\operatorname{relu}(y[1]) \\
y[l+2]=(y[l]) \text { i.e. } S=m
\end{gathered}
$$

\section{Results}

The performance of the automatic fruit disease detection model is measured by using evaluation metrics such as accuracy and multiclass cross-entropy loss function (In our case six different classes).

\subsection{Performance Analysis of DL Architectures}

All the models were trained on the augmented dataset for 220 epochs, batch size 16 , and learning rate 0.001 . Table 1 shows the performance of various CNNs based architecture on the augmented dataset. ResNet50 achieved $97.92 \%$ test accuracy which is better than ResNet18 (87.5\%) and InceptionV3 (78.75\%). The learning curve shows that the InceptionV3 model is overfitted for the present study dataset. Multiclass cross-entropy loss value is in the range of 0.0 to 1.0. Loss is the sum of all values of actual value multiplied by $\log$ of predicted value and its unit isnats. ResNet50 has given a minimum test loss of 0.0735 which indicates the model is a fast learner and learns all the parameters quickly and updates weights accordingly. ResNet18 gave a test loss of 0.213 which indicates a good learner. On the other side, InceptionV3 has given a test loss of 0.939 which revealed that this model is a poor learner.

Experimental result shows that ResNet50 is the most suitable $\mathrm{CNN}$-based architecture to improve the fruit disease detection and classification accuracy on real field-collected pomegranate image dataset. As symptoms of some diseases are not well defined therefore ResNet50 was able to give $97.92 \%$ correctness in the detection and classification of diseases and $2.08 \%$ misclassification was observed.

\subsection{Hyperparameters Tuning}

In this experiment, all the architectures were fine-tuned for 220 epochs, batch size of 16, and different learning rates on the augmented dataset (Table 3). Each model was trained for 220 epochs ( 1 epoch is when an entire dataset is passed forward and backward through the NN only once) with a batch size of 16 (The number of batches is equal to the number of iterations for one epoch). Initially few layers were frozen and then the remaining unfrozen layers were used for extracting high-level features. Each model is trained for different learning rates $(0.01,0.001$, and 0.0001 ) and the best training and test accuracy is noted for a batch size of 16 and 220 epochs. Deeper NNs are more difficult to train. Therefore, Momentum Based Stochastic Gradient Descent (SGDM) optimizer is used to improve the accuracy of the model by updating parameters on every iteration.

Table 1 Performance analysis of CNN's based architecture on the augmented data set

\begin{tabular}{|cccccccc|}
\hline \multirow{2}{*}{$\begin{array}{c}\text { Deep Learning } \\
\text { Architecture }\end{array}$} & $\begin{array}{c}\text { Image Data set Size } \\
\text { (Real field-collected data) }\end{array}$ & $\begin{array}{c}\text { Image Dataset Size } \\
\text { (After Data Augmentation) }\end{array}$ & \multicolumn{2}{c|}{ Accuracy (\%) } & \multicolumn{2}{c|}{ Cross-Entropy Loss } \\
\cline { 4 - 8 } & 1,493 & 12,750 & 95.26 & 87.5 & 0.411 & 0.213 \\
\hline Resnet-18 & 1,493 & 12,750 & 98.37 & 97.92 & 0.312 & 0.0735 \\
\hline Resnet-50 & 1,493 & 12,750 & 97 & 78.75 & 0.19 & 0.939 \\
\hline Inception-V3 & & & & & Testing & Training & Testing \\
\hline
\end{tabular}

Journal of Experimental Biology and Agricultural Sciences http://www.jebas.org 
Table 2 DL model training and hyperparameter fine-tuning

\begin{tabular}{|cccc|}
\hline Hyper Parameters & Resnet-50 & Resnet-18 & Inception-V3 \\
\hline Image input size & $224 \times 224 \times 3$ & $224 \times 224 \times 3$ & $299 \times 299 \times 3$ \\
\hline Batch Size & 16 & 16 & 16 \\
\hline Number of Epoch & 220 & 220 & 220 \\
\hline Optimizer & SGD-M & SGD-M & 0.9 \\
\hline Momentum & 0.9 & $0.01,0.001,0.0001$ & $0.01,0.001,0.0001$ \\
\hline Learning Rate & $0.01,0.001,0.0001$ & &
\end{tabular}

Table 3 Performance analysis of various CNNs based architecture after hyperparameter tuning

\begin{tabular}{|c|c|c|c|c|c|c|c|}
\hline $\begin{array}{l}\text { Deep Learning } \\
\text { Architecture }\end{array}$ & $\begin{array}{c}\text { Batch } \\
\text { Size }\end{array}$ & $\begin{array}{l}\text { No. Of } \\
\text { Epoch }\end{array}$ & Learning Rate & $\begin{array}{c}\text { Training } \\
\text { Accuracy (\%) }\end{array}$ & $\begin{array}{c}\text { Testing } \\
\text { Accuracy (\%) }\end{array}$ & Train Loss & Test Loss \\
\hline \multirow{3}{*}{ Resnet-18 } & \multirow{3}{*}{16} & \multirow{3}{*}{220} & 0.01 & 92.53 & 78.75 & 0.456 & 1.079 \\
\hline & & & 0.001 & 95.26 & 87.5 & 0.411 & 0.213 \\
\hline & & & 0.0001 & 93.92 & 81.25 & 0.46 & 0.285 \\
\hline \multirow{3}{*}{ Resnet-50 } & \multirow{3}{*}{16} & \multirow{3}{*}{220} & 0.01 & 93.92 & 90 & 0.447 & 0.146 \\
\hline & & & 0.001 & 98.37 & 97.92 & 0.312 & 0.0735 \\
\hline & & & 0.0001 & 95.14 & 93.95 & 0.379 & 0.109 \\
\hline \multirow{3}{*}{ Inception-V3 } & \multirow{3}{*}{16} & \multirow{3}{*}{220} & 0.01 & 95.486 & 72.5 & 0.258 & 1.012 \\
\hline & & & 0.001 & 97 & 78.75 & 0.19 & 0.939 \\
\hline & & & 0.0001 & 0.96875 & 65 & 0.265 & 1.0387 \\
\hline
\end{tabular}

The optimized ResNet50 model achieved $97.92 \%$ test accuracy on a batch size 16 , the number of epoch 220 , and the learning rate 0.001 . Therefore, it is an appropriate model for the present study dataset.

\section{Discussion}

There are several experiments carried out on plant diseases management using Convolutional Neural Networks (CNNs). Ahmad et al. (2020) proposed an effective CNN-based disease detection framework in plum under real field environment. The author compared four CNN-based architectures - AlexNet, VGG16, Inception-V1, and Inception-V3 for Plum diseases identifications. The results obtained from the Inception-V3 model were most encouraging with $92 \%$ accuracy. Das et al. (2020) developed a deep learning approach for automated feature selection to predict rice leaf diseases to improve crop production. $\mathrm{CNN}$ is used for automated feature engineering while PCA is used for dimensionality reduction to remove the redundant features and the diseases are classified using various classifiers. Al-Amin et al. (2019) developed an automated system for predicting potato disease using Image processing and Deep CNN Learning techniques. Publicly available (Plant Village) dataset of 2,250 potato leaves images were considered for detection and obtained
98.33\% of accuracy. Ferentinos (2018) introduced VGG - CNN model for plant leaves disease classification, detection, and diagnosis using an open image dataset of 87,848 plant leaves. The proposed system attained $99.53 \%$ accuracy and can be extended for real field disease detection. Hong et al. (2020) implemented a system to detect 9 different tomato diseases and pests using five different transfer learning models. The model was given an overall satisfactory performance. Selvaraj et al. (2019) developed an AI-based banana disease detection model using Deep Convolution Neural Networks. Dataset consists of 18,000 field images and the model has achieved $90 \%$ accuracy.

In this research work, we have collected real field images of pomegranate fruits and leaves. The most occurring diseases on pomegranate are identified and with the help of domain experts, these images are classified into six different classes. The data augmentation was performed to increase the size and diversity of the dataset. For experimentation, the dataset has been divided into $80 \%$ train and $20 \%$ test dataset. The $\mathrm{CNN}$-based models were trained on the augmented dataset to automatically detect pomegranate diseases. The proposed method was correctly detected healthy and diseased fruits along with the type of diseases. The detailed research methodology was explained from collecting the images, data augmentation, DL model selection and 
training, model performance analysis using various evaluation measures and hyperparameters tuning. Experimental outcomes prove the Inception V3 model achieved 97\% training accuracy. But it performs poorly on the test dataset and achieves only $78.75 \%$ test accuracy. Inception V3 model fails to generalize well from training dataset to test dataset. This shows the model is overfitted and not suitable for our collected dataset. However, ResNet50 achieved the highest train $(98.37 \%)$ and test accuracy $(97.92 \%)$ and minimize the test loss up to 0.0735 . It shows ResNet50 is the perfect model for our collected dataset. Further, the "Black Box" problem of DL models can be solved by integrating ResNet50 with the Grad-CAM model (Selvaraju et al., 2019).

\section{Conclusions}

This research work is mostly emphasis on area-specific research. Pomegranate fruits are being affected by unbalanced climatic conditions leading to reduce fruit yield. Further, matching fruits supply with demand becomes challenging with increasing population and decreasing natural resources. The Deep Learning technique has the potential to improve disease detection accuracy. Most of the researchers used publicly available datasets (Plant village) for pomegranate disease diagnosis. For this research, we collected stage-wise disease development real field fruits and leaves images and the most prominent five different diseases (bacterial blight, anthracnose, fruit spot, wilt, fruit borer) are considered for experimentation. CNN-based Resnet18, ResNet50, and Inception-V3 architectures have been implemented for improving disease detection accuracy. The optimized ResNet50 architecture shows superior performance with $97.92 \%$ accuracy. In the future, to deal with CNN 'Black Box' problem Grad-CAM model can be integrated with Deep Learning architectures.

\section{Acknowledgements}

The authors would like to thank Dr. A.M. Navale, Professor of Plant Pathology, Mahatma Phule Krishi Vidyapeeth, Rahuri, Maharashtra for suitable guidance to proceed further in the research study, validating the dataset and results. We are also thankful to Mr. Satish Rao, Scientist / Engineer SF - ISRO for giving valuable inputs to formulate research problem definition.

\section{Conflict of interest}

All the authors declare that there is no conflict of interest.

\section{References}

Ahmad J, Jan B, Farman H, Ahmad W, Ullah, (2020) ADisease Detection in Plum Using Convolutional Neural Network under True Field Conditions. Sensors 20: 5569. DOI: https://doi.org/10.3390/s20195569.
Al-Amin M, Bushra TA, Hoq MN (2019) Prediction of Potato Disease from Leaves using Deep Convolution Neural Network towards a Digital Agricultural System. 1st International Conference on Advances in Science, Engineering and Robotics Technology, (ICASERT 2019). DOI:10.1109/ICASERT.2019.8934933.

Das A, Mallick C, Dutta S. (2020) Deep Learning-Based Automated Feature Engineering for Rice Leaf Disease Prediction. In: Das A, Nayak J, Naik B, Dutta S, Pelusi D. (Eds.) Computational Intelligence in Pattern Recognition. Advances in Intelligent Systems and Computing, vol 1120. Springer, Singapore. https://doi.org/10.1007/978-981-15-2449-3_11

Deng J, Dong W, Socher R, Li L, Li K, Fei-Fei L, (2009) ImageNet: A large-scale hierarchical image database. IEEE Conference on Computer Vision and Pattern Recognition, Pp. 248255. DOI: https://doi.org/10.1109/CVPR.2009.5206848.

Ferentinos KP (2018) Deep learning models for plant disease detection and diagnosis. Computers and Electronics in Agriculture 145:311-318.

Hassan SM, Maji AK, Jasinski M, Leonowicz Z, Jasinska E (2021) Identification of Plant-Leaf Diseases Using CNN and TransferLearning Approach. Electronics 10: 1388. DOI: https://doi.org/10.3390/ electronics10121388.

He K, Zhan X, Ren S, Sun J (2015) Deep Residual Learning for Image Recognition. 2016 IEEE Conference on Computer Vision and Pattern Recognition (CVPR), pp. 770-778. DOI: 10.1109/CVPR.2016.90.

Hong H, Lin J, Huang F (2020) Tomato Disease Detection and Classification by Deep Learning. 2020 International Conference on Big Data, Artificial Intelligence and Internet of Things Engineering (ICBAIE), pp. 25-29. doi: 10.1109/ICBAIE49996.2020.00012.

Hossain MM, Purohit N, Sharma R, Bhattacharya S (2020) Suicide of a farmer amid COVID-19 in India: Perspectives on social determinants of suicidal behaviour and prevention strategies. DOI: https://doi.org/10.31235/osf.io/ekam3.

Kandylis P, Kokkinomagoulos E (2020) Food Applications and Potential Health Benefits of Pomegranate and its Derivatives. Foods 9 (122): 1-21, DOI: https://dx.doi.org/10.3390\%2Ffoods9020122.

Krizhevsky A, Sutskever I, Hinton G (2012) ImageNet Classification with Deep Convolutional Neural Networks. Communications of the ACM 60 (6): 84-90. https://dl.acm.org/doi/10.5555/2999134.2999257.

Liu M, Chen L, Du X, Jin L, Shang M (2021) Activated Gradients for Deep Neural Networks. IEEE Transactions on Neural 
Networks and Learning Systems, Pp. 1-13, DOI: 10.1109/TNNLS.2021.3106044.

Melgarejo-Sánchez P, Núñez-Gómez D, Martínez-Nicolás J, Hernández F, Melgarejo P (2021) Pomegranate variety and pomegranate plant part, relevance from bioactive point of view: a review. Bioresources, Bioprocess, 8, 2. DOI https://doi.org/ 10.1186/s40643-020-00351-5.

OECD/FAO (2016) International Regulatory Co-operation and InternationalOrganisations: The Case of the Food and Agriculture Organization of the United Nations (FAO), OECD and FAO.

Reddy TK, Dutta M (2018) Impact of Agricultural Inputs on Agricultural GDP in Indian Economy. Theoretical Economics Letters 8: 1840-1853.

Rehman F, Naz N, Kalsoom M (2021) Plant Diseases and Food Security: An Overview. Agrospheres:e-Newsletter 2 (1): 29-31.

Russakovsky O, Deng J, Su H, Krause J, Satheesh S, Ma S, Huang Z, Karpathy A, Khosla A, Bernstein M, Berg AC, Fei-Fei L (2015) ImageNet Large Scale Visual Recognition Challenge. International Journal of Computer Vision 115: 211-252. DOI: https://doi.org/10.1007/s11263-015-0816-y.

Sarker IH (2021) Deep Learning: A Comprehensive Overview on Techniques, Taxonomy, Applications and Research Directions. SN Computer Science 2(6):420. DOI: https://dx.doi.org/ 10.1007\%2Fs42979-021-00815-1.

Selvaraj MG, Vergara A, Ruiz H, Safari N, Elayabalan S, Ocimati $\mathrm{W}$, Blomme G (2019). AI-powered banana diseases and pest detection. Plant Methods 15: 92. DOI https://doi.org/10.1186/s13007-019-0475-z.

Selvaraju RR, Cogswell M, Das A, Vedantam R, Parikh D, Batra D(2019) Grad-CAM: Visual Explanations from Deep Networks via
Gradient-based Localization. International Journal of Computer Vision, Volume-128, Pp. 336-359, https://arxiv.org/abs/1610.02391.

Shorten C, Khoshgoftaar TM (2019) A survey on Image Data Augmentation for Deep Learning. Journal of Big Data 6, Article number: 60 (2019). DOI: https://doi.org/10.1186/s40537-0190197-0.

Simonyan K, Zisserman A (2015) Very Deep Convolutional Networks for Large-Scale Image Recognition. The 3rd International Conference on Learning Representations (ICLR2015). Available at https://arxiv.org/abs/1409.1556 access on 29th April 2021.

Szegedy C, Vanhoucke V, Ioffe S, Shlens J (2016) Rethinking the Inception Architecture for Computer Vision. 2016 IEEE Conference on Computer Vision and Pattern Recognition (CVPR): 2818-2826, doi: 10.1109/CVPR.2016.308.

Taylor L, Nitschke G (2018) Improving Deep Learning with Generic Data Augmentation. IEEE Symposium Series on Computational Intelligence (SSCI), Pp. 1542-1547, DOI: https://doi.org/10.1109/SSCI.2018.8628742.

Wang J, Lan C, Liu C, Ouyang Y, Zeng W, Qin T (2021) Generalizing to Unseen Domains: A Survey onDomain Generalization. International Joint Conference on Artificial Intelligence (IJCAI-21), Pp. 1-15. DOI: https://doi.org/10.24963/ijcai.2021\%2F628.

Yadav DB, Sanap DJ, Pokharkar VG (2020). Total Factor Productivity Growth in Pomegranate Crop of Maharashtra. Indian Journal of Agriculture Business 6 (1): 9-14. DOI: http://dx.doi.org/10.21088/ijab.2454.7964.6120.1.

Zeiler M., Fergus R (2014) Visualizing and Understanding Convolutional Networks. Computer Vision - ECCV 2014. Lecture Notes in Computer Science 8689: 818-833, Springer, Cham. https://doi.org/10.1007/978-3-319-10590-1_53. 